\title{
How to motivate for correct sampling projects based on costs and benefits of fit-for-purpose sampling solutions
}

\section{Trevor Bruce ${ }^{\mathrm{a}}$ and Richard C.A. Minnitt}

${ }^{a}$ FLSmidth, Boksburg East, 1459, South Africa

bVisiting Emeritus Professor, University of the Witwatersrand, Johannesburg, South Africa

\section{Editor's summary}

Mining companies are generally reluctant to install high-cost sampling systems at operations that have been "functioning well" for many years. The principal objection to installing new equipment to extract correct and representative samples in process flows is the time and costs involved. The Theory of Sampling (TOS) provides a structured framework for identifying and quantifying the errors and bias associated with any sampling event, but this may often be insufficient to motivate for investment in new, correctly designed sampling equipment. Financial losses arising from substandard sampling installations are usually disregarded because valueadded economic benefits from good quality sampling solutions are most often invisible, while the adverse cost from inappropriate systems are plainly obvious. Depending on the specific needs, a Fit for Purpose (FFP) sampling solution may be acceptable, provided the magnitude of sampling errors is understood and assay results are interpreted accordingly. Categorical levels of acceptable accuracy and precision can be established depending on the sampling position in the mining value chain and the nature of the decisions to be made. Objective benefits of proposed FFP sampling solutions must be presented to relevant decision

DOI: $10.1255 /$ sew.2021.a45
(C) 2021 The Authors
Published under a Creative Commons
BY-NC-ND licence
CC $\$$ BY N

makers in such a way that adverse subjective decisions based on only poorly resolved economic information are made difficult (impossible). Examples of benefits from motivating such implementation of FFP sampling solutions at sampling facilities around the world, are presented.

\section{Introduction}

Humans rationalise differently based on individual cognitive processes or reasoning (culture, experience, educational background, management level). Decision-makers in mining financial departments may rationalise very differently about spending funds on corrective sampling compared to technical sampling experts, meaning that well-conceived corrective sampling projects could be rejected. Irrespective of the appropriateness of the technical design, or imperatives of the Theory of Sampling (TOS), approval for implementing a corrective sampling solution is declined unless value-added benefits can be defined and corroborated with numerical proof. The fact that the costs of poor sampling never show up as a line item in annual financial statements means managers do not see a figure that represents a loss to their earnings. Attitudes towards meaningful expenditure on correctly designed and installed sampling equipment, therefore, remain obstinate. The rationale for the implementation of improved sampling solutions must be presented as simply as possible leaving little room for subjective interpretation.

Estimating added value from implementing sound TOS-based sampling practices does not require a long or complicated report. A simple "back to basics" approach, pointing out the critical

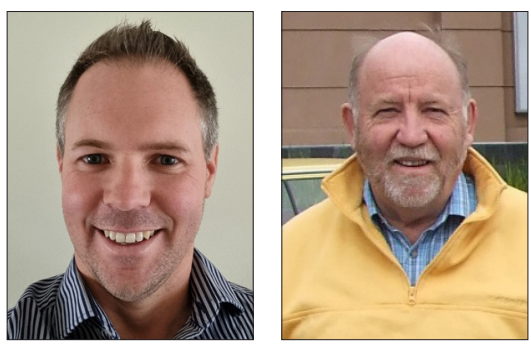

points and findings with a summary of the financial benefits provides a much better chance of getting your point across. The "sampling fraternity" should also acknowledge that implementation of the "close to perfect sampling solutions" are not always feasible. The need to make compromises, knowing that not all solutions are perfect, may still provide some added value by reducing sampling errors compared to current operations.

Here is a look at a back-to-basics approach for demonstrating the benefits of installing corrective sampling protocols and equipment to those approving budgets and to reluctant shareholders.

\section{General concepts}

Several observations have come to light as a result of presenting appeals to the boards of companies for funding to improve sampling facilities.

\section{Distrust of large unsubstantiated numbers}

The adage that "If something sounds too good to be true, then it probably is" is still applicable. Statements that say "... better sampling can produce an additional US $\$ 25,000,000$ worth of on-grade ore per annum..." sound too good to be true and arouse a certain level of scepticism in the listeners. Numbers this large appear unrealistic and are probably not trustworthy unless they can be validated by realistic examples. It is better to begin by offering small numbers, for example "...correct sampling can deliver an additional US\$7500 for a 100,000t shipment of iron ore..." and allow the decision makers do the mental arithmetic themselves. Producers who, for example, deliver over 3400 shipments of this size per year can easily see the potential value of better sampling. 
Disinterest in statistics without practical application

Technical statements with equations and figures could be lost on non-technical managers and executives. What they would prefer to hear is "... let me show you what improving your Sampling, Preparation and Measurement (SPM) can do for your profits."

Executive committees have neither the time nor the interest in detailed or complex calculations of precision that technical jargon alone will seldom motivate budget approval. The insight, interest and understanding of sampling technicalities is not common amongst such audiences, but simple statements such as "...every $0.01 \%$ improvement on the SPM precision could potentially increase the on-grade production by $0.01 \%$..." are more likely to pique their interest long enough for budget approval.

\section{Penalties for out-of-spec products}

The cost of underestimating the target analyte content is not reported in financial statements as it is not quantifiable, and in many cases misunderstood. What should be seen on a company's financials are the costs incurred due to penalties paid for delivering off-spec grade ore and deleterious elements that exceed allowable limits. Quantifying the amount paid in penalties highlights a quantifiable "real cost" that could be reduced by implementing better sampling practices and systems.

\section{Names mean more than numbers}

The role and responsibility of financial managers may very likely not allow them the privilege of time to digest the theoretical aspects of the TOS. Collaboration and certification of designs by well-known reputable experts add greater confidence to improved sampling solutions than complex formulas. Recruiting specialists who are able to validate or verify decisions about proposed sampling installations is an effective method of convincing project owners and shareholders that improved sampling will be money well spent.
Few are impressed by jargon and equations

Correct sampling nomenclature and application of equations to estimate financial benefits is critical, but shareholders and financial managers are unimpressed with complex procedures and formulas. Shareholders are much more likely to approve projects provided one can demonstrate that the estimated added value through improved sampling has been validated by reputable persons or sources.

\section{Fit for purpose sampling}

Providing correct sampling solutions is a high-cost exercise. Inevitably the more stringent project owners are about installing correct sampling solutions, the higher the capital costs will be. This article does not dispute the fact that samples cannot be trusted if the principles of the TOS are not followed. The rational man strives for perfection, but knowing that things in life are rarely perfect, this article wants to demonstrate that "inferior sampling" may sometimes still offer value. Of course, the value depends on how the resulting analysis and associated confidence intervals will be used. This also assumes that benefits are not outweighed by the cost of taking, transporting, preparing and assaying the sample-a simple concept, but one that may in fact be overlooked by the sampling fraternity.

\section{ISO standards vs the TOS}

"ISO compliant" is a term often used when discussing upgrades to sampling facilities. ISO standards, although critical in establishing standard methods of sampling, preparation and analysis for various commodities and materials, are too often used out of context! There appears to be a widespread misunderstanding that compliance with a relevant ISO standard will automatically result in the best possible sampling results. ISO standards should be looked on as recommendations to be used to assist a facility in ascertaining a minimum precision and level of confidence in accordance with international standards. However, improving sampling equipment and methods above the minimum requirement recommended by
ISO standards, can indeed add further value to a producer. As an example, if iron ore producers reduce the precision on a 270,000 tonne lot, below the $\beta_{\mathrm{SPM}}{ }^{\mathrm{a}}$ of $0.34 \%$ Fe stipulated by ISO 3082, substantial improvements in on-grade ore production can be achieved.

Compliance is one thing, but before setting a limit on the resources assigned to sampling and analysis, it is critical to establish the monetary value of each sample using a minimum requirement, compared to what it could be worth if additional resources were spent on improving it. Rather than strict compliance with minimum requirements from relevant ISO standards, other aspects, especially efficiency for operational purposes, should be considered when specifying a sampling point. For example, taking a higher number of primary increments, and analysing more subsamples than required by ISO during the loading of an iron ore vessel can give a better indication of the grade during loading. This can then be used to blend the product mixture that goes into the vessel more efficiently.

Although some principles may be the same it is definitely unwise to apply guidelines from a single ISO standard to different materials. For example, the ISO 3082 for iron ores should not be used to estimate sampling compliance of phosphate fertilisers, because the material characteristics are in fact detrimentally different; in this case the proper material-specific standard must be applied. Where no specifications are available for a material, TOS practices such as heterogeneity tests and sampling calibration for establishing nomograms should be applied, although the work efforts (costs) are never small. Explaining the need for, and the workings of, a full-scale industrial heterogeneity test is no small feat in the board room.

\section{Motivating a sampling project} The objective of a sampling study should be to demonstrate how improved sampling practices can improve profitability. Motivating a project to improve

${ }^{a}$ Overall precision for Sampling Preparation and Measurement $\beta_{\mathrm{SPM}}=2 \sigma_{\mathrm{SPM}}$. 
sampling equipment and protocols requires an understanding of the complete value chain so that expectations about the outcome of the sampling project are met. This may include defining the levels of reliability and precision to be achieved as a result of improvements to sample extraction, sample handling/transport, sample preparation and measurement. Motivating for added value and increased profit from improved sampling precision must be supported by realistic estimates of the capital cost and required investment. The capital investment needed for taking better samples using better sampling equipment, ensuring the correct sample size and sample frequency, must be calculated accurately. In addition, the costs of ensuring samples are correctly transported, prepared, analysed and reported, within an acceptable turn-around time that allows real-time changes to be made, must be established. The total investment required in terms of time, money and effort must be understood by managers and financial officers, if proposals to improve sampling processes are to have credibility and integrity. The skills of producing a convincing business case should be on everybody's agenda, sampling experts no exception.

\section{Case studies and examples}

Rapid turn-around vs high precision A smelter aimed to get less than 5ppm precious metal in slag which it sells as silica waste for US\$3/tonne. Slag analyses over a six-month period indicated the precious metal content to be as high as $18 \mathrm{ppm}$. The operating costs to recycle slag through the furnace, after it has cooled, is approximately US\$5700/ tonne, so although the sampling error could be reduced by improved sampling methods, the cost-benefit would be minimal unless the samples can be collected and analysed before the slag is cast. On average around 10 tonnes of slag is produced per cycle, meaning that the value of precious metals in a single slag cycle would have to be greater than US $\$ 57,000$ to make re-cycling through the furnace feasible. If samples can be collected and analysed before the slag is poured, then remedial actions such as increasing the residence time in the furnace can be performed. In this case precision of the result is not as crucial as the sampling-to-analysis turn-around time so this is where the focus for the project was placed, and rightly so. But if the furnace charge is analysed before smelting it is possible to modify the charge and reduce the risk of precious metals reporting to the slag. In this case the precision is more critical to the process as small variations in the charge can affect the smelt efficiency.

Reducing the loss of precious metals in the slag to less than 5ppm would result in an additional precious metal production of over US\$400,000 per annum. This figure, based on historical data gathered from the smelter, compares the actual gold content of processed slag with what it should have been if the Au grade was maintained below 5 ppm. Assuming a three-year return on investment, a sampling solution costing less than US $\$ 1,200,000$ would indeed add value. This example illustrates the necessity of understanding the economic and logistical implications, limitations and the effect of improved sampling before proposing a sampling-to-analysis solution.

\section{A less than perfect sampling solution may still add value}

A less than perfect sampling solution was observed at a phosphate fertiliser production plant where phosphate slurry is mixed with $\mathrm{H}_{2} \mathrm{SO}_{4}$ in a reactor vessel to produce phosphoric acid. Dip samples are taken between $50 \mathrm{~cm}$ and $500 \mathrm{~cm}$ below the slurry surface (sampling experts would collectively frown severely!). Concentrations of free sulphate, measured by titration, and phosphoric acid cannot be quantified in the complete vessel with any certainty using this imperfect sample type, but some knowledge of the concentrations is critical for controlling processes in the reactors.

In this case, time is of the essence. It is critical that the sample be filtered and analysed as soon after extraction as possible because the reaction in the sample container continues as the sample cools, affecting the analytical results significantly. To improve the integrity of the sample, an insulated sample container should be used to collect the sample (Craig Ritchie, 2018, personal communication). In addition, rapid transport to the laboratory using a pneumatic air tube conveyance was strongly suggested.

\section{Too many compromises invalidate} the sampling point

When faced with tight deadlines and minimal budgets, project managers and engineers often must make compromises in correct sampling to complete a project. Although some compromise is almost always required, too many can completely invalidate the sampling solution being used. For each compromise made, the total effect on the result should be established to understand the ultimate financial impact on the plant accountings. This is also where the project owners must know what is needed as a minimum to achieve the required sampling precision and to meet all the operational requirements to ensure that the sampling solution providers they are using do not lead them astray. Using internal resources or external specialists to define what is needed for sampling installations is one method to achieve this, which is a lot more cost effective than attempting to investigate and repair "what went wrong" after the system has been implemented.

\section{Good sampling to analytical practices}

At critical operations, such as port loading facilities, correct sampling together with rapid sample preparation and measurement can have massive financial rewards for the company.

As an example of this, in a welldesigned iron ore port facility every reasonable effort was made to minimise SPM errors in the facility. This, together with the addition of automated sample preparation and analysis, not only makes the system more precise but also offers the value of rapid preparation and analysis. For this facility, even though the sampling building did not have enough head room to fit a standard traditional cross-stream sampler, an 


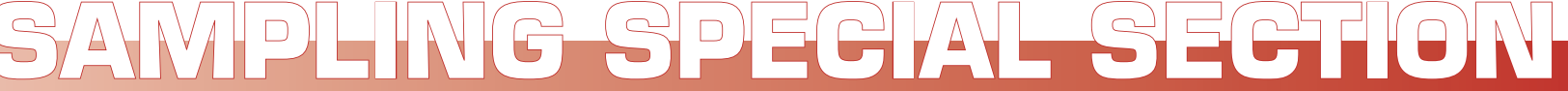

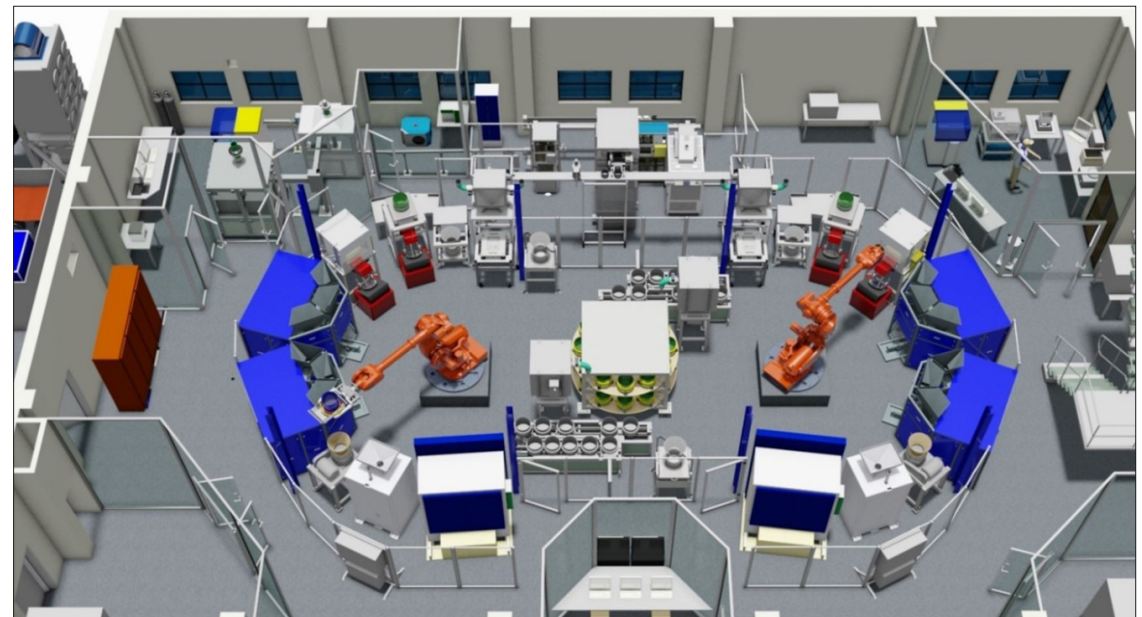

Figure 1. Robotic automation for sampler preparation and analysis for chemical, PSD and moisture conveyor.

innovative alternative was developed (which involved a long radius swing arm which crossed the stream and lifted the sample material to the floor above). This key contribution required that both the client and supplier had a strong understanding of the sampling requirements and worked together to find a feasible solution. The result of this is a turnaround time on results of less than $6 \mathrm{~h}$ after the completion of the ship being loaded, with precision of less than $0.15 \%$.

\section{Value of investment}

The iron ore producer has not released the actual value of the investment to make this facility perform as it does today, but the following available information will suffice.

1) The final analysis is available $6 \mathrm{~h}$ after ship loading is complete; this was previously a minimum of $48 \mathrm{~h}$. The benefit is that the lot can be invoiced nearly two days earlier than before, resulting in an estimated gain of US\$5500 per 270,000 ton shipment on interest alone.

2) For large lots, the laboratory can release data during ship loading with a precision of less than $0.15 \%$. This data is used to adjust the blend between low- and high-grade ore to control the final blended grade being loaded.

3) The iron ore producer can use the high precision as proof of the quality of its product thereby giving them a commercial advantage over their competition (bragging rights).

4) Reduced risk of penalties due to deleterious elements exceeding upper specification limits of the lot, or lower than specified Fe content.

5) Disputes over the quality of the ore loaded through this terminal are quickly settled due to the overall compliance of the complete facility with the relevant laboratory and material specifications.

This plant has proven that rapid analysis with consistently high precision is possible, so the producer is currently investigating upgrades and expansions of this facility to increase sampling and analytical capacity with the aim of optimising in-ship grade blending.

\section{Conclusions}

A full understanding of the complete mineral production and sales process before and after the sampling point is essential. Such insights allow one to appreciate the current usage of the sampling results, as well as identify other potential uses and added value opportunities, especially if the quality of the sampling can be improved. Ascribing a monetary value to a sample in a process or procedure is an important asset. An understanding of the levels of precision or sampling correctness of a sample, as well as the turn-around time to analysis will influence the value. An appreciation of various constraints, such as applicable standards or specifications, physical space available, accessibility for inspections, maintenance and sample collection, plant down time available for the installation, distance of sampling point to the laboratory must all be taken into consideration to create a fit-for-purpose sampling solution.

In the process control domain, fit-forpurpose sampling has a wider scope as compromises can be made in various aspects of sampling to suit the application, provided the consequences of these compromises are understood. In the product control domain, where the same compromises cannot be made, fitfor-purpose sampling can still be applied when considering how the sampling will affect other aspects of the facility such as material throughput and the value of faster sampling-to-analysis. Using a crossstream sampler will not affect loading rates, whereas a stop belt sampler will; therefore, the cross-stream sampler is fitfor-purpose in this case.

The feasibility of motivating and actioning an upgrade process required by a facility to exploit the expected valueadd from the specified sampling solution also requires insight and understanding. Some of the actions can include automated sample collection and transport systems to increase turn-around time from sampling to laboratory. Other actions could include upgrades to the laboratory to accommodate the sampling solution and an upgrade to standard operating procedures. The result of such actions would enable the facility to respond to improved sample information to achieve added value possibilities.

It is important that project owners communicate their needs to sampling equipment suppliers in a way that ensures the supplier provides the best solution for their application. Lowest cost procurement should not govern the choice and selection of critical success equipment, of which sampling equipment range very high, as this is likely to turn out to be a costly error of judgement. A policy of lowest cost procurement is short-sighted as it may mean not only inferior equipment selection, but also incorrect sample extraction that 


\section{SAMULANE SPEGLAL SEGHEIN}

introduces costly bias associated with poor sampling.

The greatest deterrent of decisions to install appropriate or improved sampling solutions is that the benefits are invisible, while the adverse costs are obvious. No financial statements tell CEOs what the benefits from proper, FFP or indeed fully representative sampling are. The only indications might be increases in the costs of reagents and consumables. When presenting a sampling solution to an executive committee, with the hope of getting approval, the primary motivational factor should be a clear demonstration of the monetary benefits the solution can offer. The basis of the motivation should be a comparison of cost-to-implement against the longterm expected returns on investment. All mathematical and other technical aspects of the proposed solution should be kept as straightforward as possible with only key results presented, but which are verified by a well-respected specialist in the field.

\section{Caveat}

Of course, there always also is the option of being able to explain adequately more of the essential technicalities in a manner that is fully understandable, so that management, CEO's board members, investors ... actually gain an increased factual knowledge. This will always be part of a best business case. This challenge still leaves room for the diligent, competent, didactically motivated fraction of the international community of sampling experts, who not all necessarily need to rush off to get a MBA degreeteam collaboration will always go a long way!

\section{The ideal management commitment}

The reader is referred to an earlier Sampling Column addressing the key issue of awareness and commitment on the part of management: Theory of Sampling-an approach to representativity offering front line companies added value and potential substantial savings.

F. Rendeman, J.R. Pedersen and K.H. Esbensen, "Theory of Sampling-an approach to representativity offering front line companies added value and potential substantial savings", Spectrosc. Europe 32(3), 23-26 (2020). https://doi.org/10.1255/ sew.2020.a 1 